\title{
Discovery of the supernova remnant G351.0-5.4 ${ }^{\star}$
}

\author{
F. de Gasperin ${ }^{1}$, C. Evoli ${ }^{2}$, M. Brüggen ${ }^{1}$, A. Hektor ${ }^{3}$, M. Cardillo ${ }^{4}$, P. Thorman ${ }^{5}$, \\ W. A. Dawson ${ }^{5,6}$, and C. B. Morrison ${ }^{5,7}$
}

\author{
1 Universität Hamburg, Hamburger Sternwarte, Gojenbergsweg 112, 21029 Hamburg, Germany \\ e-mail: fdg@hs . uni-hamburg.de \\ 2 Institut für Theoretische Physik, Universität Hamburg, Luruper Chaussee 149, 22761 Hamburg, Germany \\ 3 National Institute of Chemical Physics and Biophysics, Ravala 10, 10143 Tallinn, Estonia \\ 4 INAF-Osservatorio di Arcetri, Largo Enrico Fermi 5, 50125, Firenze, Italy \\ 5 University of California, One Shields Avenue, Davis CA 95616, USA \\ ${ }^{6}$ Lawrence Livermore National Lab, 7000 East Avenue, Livermore CA 94550, USA \\ 7 Argelander Institute for Astronomy, University of Bonn, Auf dem Hügel 71, 53121 Bonn, Germany
}

Received 12 May 2014 / Accepted 31 July 2014

\begin{abstract}
While searching the NRAO VLA Sky Survey (NVSS) for diffuse radio emission, we have serendipitously discovered extended radio emission close to the Galactic plane. The radio morphology suggests the presence of a previously unknown Galactic supernova remnant. An unclassified $\gamma$-ray source detected by EGRET (3EG J1744-3934) is present in the same location and may stem from the interaction between high-speed particles escaping the remnant and the surrounding interstellar medium. Our aim is to confirm the presence of a previously unknown supernova remnant and to determine a possible association with the $\gamma$-ray emission 3EG J17443934. We have conducted optical and radio follow-ups of the target using the Dark Energy Camera (DECam) on the Blanco telescope at Cerro Tololo Inter-American Observatory (CTIO) and the Giant Meterwave Radio Telescope (GMRT). We then combined these data with archival radio and $\gamma$-ray observations. While we detected the extended emission in four different radio bands $(325,1400$, 2417, and $4850 \mathrm{MHz}$ ), no optical counterpart has been identified. Given its morphology and brightness, it is likely that the radio emission is caused by an old supernova remnant no longer visible in the optical band. Although an unclassified EGRET source is colocated with the supernova remnant, Fermi-LAT data do not show a significant $\gamma$-ray excess that is correlated with the radio emission. However, in the radial distribution of the $\gamma$-ray events, a spatially extended feature is related to supernova remnant at a confidence level of $\sim 1.5 \sigma$. We classify the newly discovered extended emission in the radio band as the old remnant of a previously unknown Galactic supernova: SNR G351.0-5.4.
\end{abstract}

Key words. ISM: supernova remnants - radio continuum: ISM - gamma rays: ISM

\section{Introduction}

Supernova remnants (SNRs) have a fundamental role inside our Galaxy and in general, inside their host galaxies. The gravitational collapse of a stellar core causes the ejection of the outer layers of the star at very high velocities $\left(\approx 10000 \mathrm{~km} \mathrm{~s}^{-1}\right)$. The interaction between the circumstellar medium and the ejected matter produces shock waves propagating at a non-negligible fraction of the speed of light $(v \approx 0.1-0.2 c)$. In addition, diffusive shock acceleration (DSA) predicts that shock fronts observed in SNRs are efficient accelerators of Galactic cosmic rays (GCR). Accelerated protons interact with the protons of the ISM producing a $\pi^{0}$ meson that then decays into two $\gamma$-ray photons (Malkov $\&$ Drury 2001, and references therein). At the same time, accelerated electrons will follow a power-law momentum distribution and produce radio non-thermal emission from the SNR. The proton-proton interaction time decreases with the target density enhancement. Consequently, $\gamma$-ray emission from SNRs can be more easily seen when the remnant interacts with a target denser than the ISM, such as a molecular cloud, as is the case with S147 (Katsuta et al. 2012), IC443 (Tavani et al. 2010; Ackermann et al.

* FITS files of Figs. 1 and 5 are only available at the CDS via anonymous ftp to cdsarc.u-strasbg. fr (130.79.128.5) or via http://cdsarc.u-strasbg.fr/viz-bin/qcat?J/A+A/568/A107
2013), W28 (Giuliani et al. 2010), and W44 (Ackermann et al. 2013; Cardillo et al. 2014).

It has been predicted (Berkhuijsen 1984; Tammann et al. 1994) that the total number of Galactic SNRs is between 1000 and 10000 . However, only 274 SNRs have been identified (Green 2009, and references therein). Most of the known SNRs are sources of radio-synchrotron emission and radio observations were routinely used to discover new candidates. Images from radio surveys, such as the NRAO VLA Sky Survey (NVSS; Condon et al. 1998), were successfully used in the past to identify new SNR candidates (see e.g. Green 2001, for the case of G353.9-2.0). The NVSS provides polarized images at $1.4 \mathrm{GHz}$, covering the sky down to a declination of $-40^{\circ}$, and its data were collected with the VLA in D-configuration. Although the survey images were obtained with short snap-shots which may limit the sensitivity on the largest scales, the NVSS is a good starting point to look for extended radio emission, up to scales of $\sim 16^{\prime}$.

By inspecting NVSS radio maps, we discovered a new candidate SNR which we designated SNR G351.0-5.4. Here we present a radio follow-up at $325 \mathrm{MHz}$ using the GMRT and an optical follow-up using DECam on the CTIO $4 \mathrm{~m}$ Blanco telescope. Furthermore, SNR G351.0-5.4 appears to be co-located with the unclassified EGRET $\gamma$-ray source 3EG J1744-3934. In order to test the EGRET detection, we have conducted a search 
Table 1. Coordinates and flux densities at $325 \mathrm{MHz}$ of the subtracted point sources.

\begin{tabular}{cccc}
\hline \hline Source name & RA $(J 2000)$ & Dec $(J 2000)$ & $S_{325}(\mathrm{Jy})$ \\
\hline NVSS J174802-385754 & $17^{\mathrm{h}} 48^{\mathrm{m}} 01^{\mathrm{s}}$ & $-38^{\circ} 57^{\mathrm{m}} 51^{\mathrm{s}}$ & 0.24 \\
NVSS J174543-392912 & $17^{\mathrm{h}} 45^{\mathrm{m}} 44^{\mathrm{s}}$ & $-39^{\circ} 29^{\mathrm{m}} 12^{\mathrm{s}}$ & 0.15 \\
NVSS J174737-394957 & $17^{\mathrm{h}} 47^{\mathrm{m}} 38^{\mathrm{s}}$ & $-39^{\circ} 49^{\mathrm{m}} 59^{\mathrm{s}}$ & 0.13 \\
NVSS J174122-392935 & $17^{\mathrm{h}} 41^{\mathrm{m}} 22^{\mathrm{s}}$ & $-39^{\circ} 29^{\mathrm{m}} 35^{\mathrm{s}}$ & 0.12 \\
\hline
\end{tabular}

for $\gamma$-ray emission at the location of SNR G351.0-5.4 with the Large Area Telescope (LAT) on board the Fermi Gamma-Ray Space Telescope.

In Sect. 2 we present the GMRT (Sect. 2.1), DECam (Sect. 2.3) and Fermi (Sect. 2.4) observations and the respective data reductions. The main characteristics of SNR G351.0-5.4 are presented in Sect. 3. Discussion and conclusions are in Sects. 4 and 5 , respectively.

\section{Observations and data reduction}

\subsection{GMRT}

Using the GMRT ${ }^{1}$, we observed the radio emission in the direction of SNR G351.0-5.4 with a run of $5 \mathrm{~h}$. During the night of March 18-19, 2013, visibilities were recorded at $325 \mathrm{MHz}$ in dual-polarization (RR and LL). We used an $8 \mathrm{~s}$ integration time, and a $33 \mathrm{MHz}$ bandwidth divided into 512 channels. The primary flux and bandpass calibrator 3C 286 was observed at the start and end of each run, while the secondary gain calibrator PKS 1827-36 was observed every half an hour for three minutes. The total time-on-target was $135 \mathrm{~min}$.

The visibility data was processed using a semi-automated $\mathrm{CASA}^{2}$-based data reduction pipeline written in Python. Data were initially flagged through a series of increasingly sensitive runs of the CASA tasks FLAGDATA in the "rflag" mode. During these cycles a refined bandpass was obtained. 3C 286 was used as the flux and bandpass calibrator. Flags were then reset and, once corrected for the bandpass, AOflagger (Offringa et al. 2012) was used to detect and remove radio frequency interference. At the end of the procedure seven antennas (two of which in the core) were manually flagged together with approximately $45 \%$ of the remaining data because of interference. After an initial phase and amplitude calibration on 3C 286, bad baselines were identified and removed by inspecting the BLCAL calibration tables, and the phase and amplitude solutions were extended to the target field. Several cycles of phase self-calibration, with subsequent clipping of the outliers, were performed and a final cycle of amplitude self-calibration was eventually made. Calibration in time and amplitude in the direction of the four brightest sources in the field and subsequent subtraction ("peeling", Noordam 2004) was done iteratively after subtracting the best model of the rest of the field. The source to peel is then subtracted and the other sources restored. Location of the peeled sources and flux densities are given in Table 1.

A final image was made removing baselines longer than $8 \mathrm{k} \lambda$ and data were tapered applying a circular Gaussian taper with FWHM at $18 \mathrm{k} \lambda$ to reach a resolution of $141^{\prime \prime} \times 89^{\prime \prime}$ and enhance the extended emission of the target. The resulting primary-beam corrected image has a background noise of 1.5 mJy beam $^{-1}$ and is presented in Fig. 1. The flux densities of the east and west parts of the shell are listed in Table 2.

\footnotetext{
1 GMRT: http://gmrt.ncra.tifr.res.in

2 CASA: http://casa.nrao.edu
}

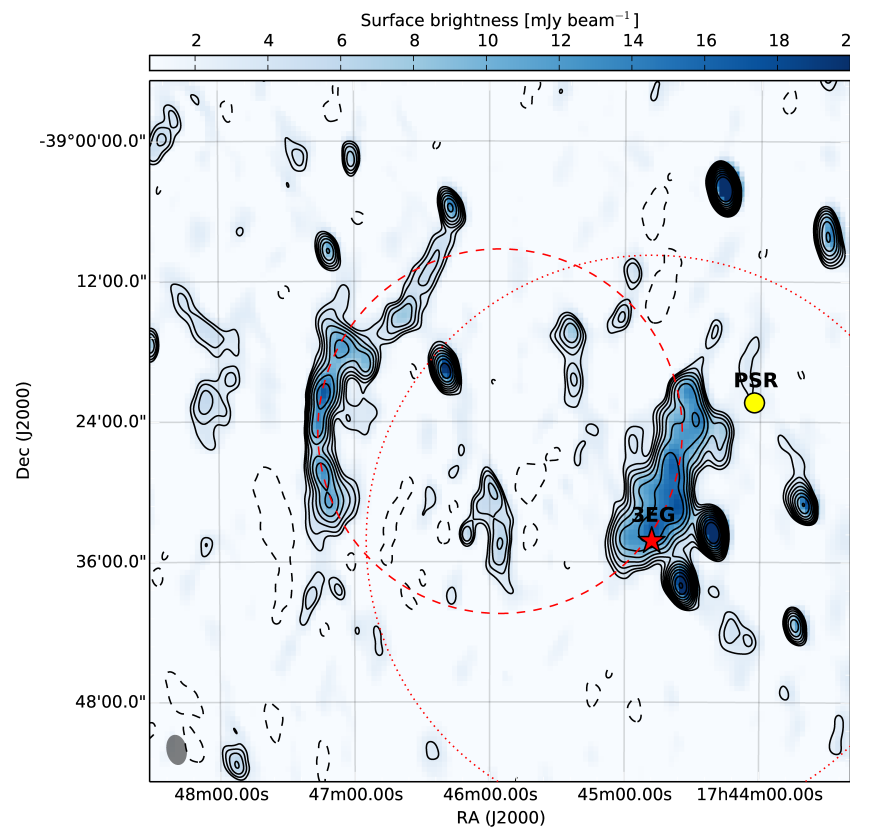

Fig. 1. GMRT radio maps of SNR G351.0-5.4 at $325 \mathrm{MHz}$. Beam size is shown in the bottom left corner, contours are equally spaced between $2 \sigma$ and $20 \sigma$ in logarithm space with $\sigma=1.5$ mJy beam $^{-1}$. Dashed contours are at $-2 \sigma$. The dashed circle traces the SNR shell location, while the yellow dot shows the positions of the pulsar PSR J1744-3922 and the red star shows the position of the $\gamma$-ray source 3EG J1744-3934. The dotted circle is the $1 \sigma$ position error of the $\gamma$-ray source.

\subsection{Archival radio data}

A map at $1400 \mathrm{MHz}$ has been extracted from the NVSS (Condon et al. 1998). The image rms is $\sim 0.5 \mathrm{mJy}^{-1}$ beam ${ }^{-1}$ at a resolution of $45^{\prime \prime}$. This high-resolution image of SNR G351.0-5.4 shows the extremely narrow front of the expanding shell towards the east (see Fig. 2a). Although the extension of the SNR is smaller than the largest detectable angular scale for the VLA in D-configuration, flux density measurements are likely to be underestimated due to the poor coverage of the central part of the $u v$-plane caused by the short observing time. NVSS polarized intensity in the direction of SNR G351.0-5.4 is too weak to provide useful information.

A map at $2417 \mathrm{MHz}$ has been obtained from the Parkes $2.4 \mathrm{GHz}$ Survey of the southern Galactic plane (Duncan et al. 2012) with an image noise of $\sim 11 \mathrm{mJy}^{\text {beam }}{ }^{-1}$ and a resolution of $10^{\prime}$ (see Fig. 2b). The target is detected but it is embedded in a larger-scale emission. Enhanced emission is visible in the corresponding regions of the shell that are detected in the higherresolution maps. Compared to all other radio maps, the Parkes $2.4 \mathrm{GHz}$ map shows entirely the extended structure of the source, having been extracted from a single-dish observation with no filtering applied. Polarized intensity maps from the Parkes $2.4 \mathrm{GHz}$ survey have also been analysed. After applying a cut at $2 \sigma$ both in the total intensity and in the polarized intensity maps, a region with a fractional polarization emission of $\sim 15 \%$ has been detected in the north-west part of the remnant (see Fig. 3).

We obtained a high-frequency radio map from the ParkesMIT-NRAO (PMN; Griffith \& Wright 1993) survey at $4850 \mathrm{MHz}$ with an image local noise of $\sim 11 \mathrm{mJy} \mathrm{beam}^{-1}$ and a resolution is of 4'.9 (see Fig. 2c). The SNR G351.0-5.4 is visible even at this higher frequency but the elongated bow structure on the east is broken into two distinct parts. The presence of an extended emission inside the boundary of the SNR is also visible. 


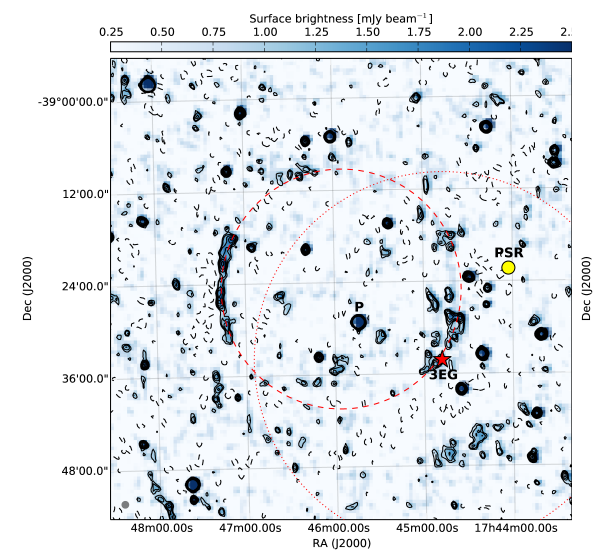

(a) VLA (NVSS) - $1400 \mathrm{MHz}$

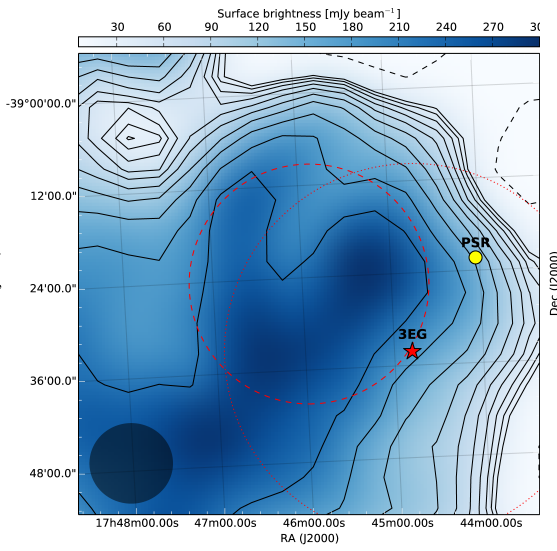

(b) Parkes $64 \mathrm{~m}-2417 \mathrm{MHz}$

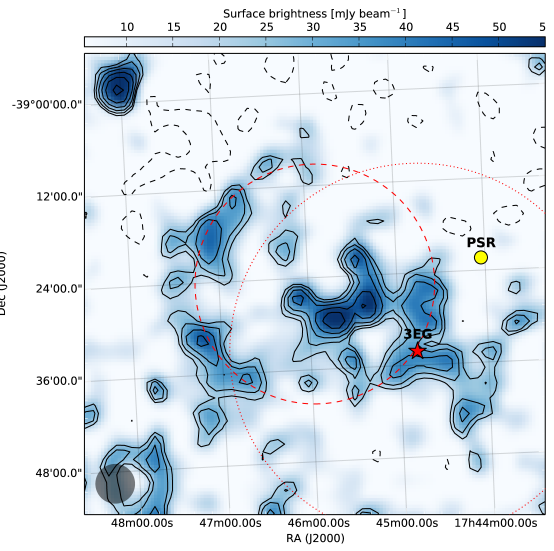

(c) Parkes 64m (PMN) - $4850 \mathrm{MHz}$

Fig. 2. Radio maps of SNR G351.0-5.4 at 1400 (NVSS), 2417 (Parkes 64m) and 4850 (PMN) MHz. Beam size is shown in the bottom left corner of every panel, contours are equally spaced between $2 \sigma$ and $20 \sigma$ in logarithm space with $\sigma_{1400}=0.5 \mathrm{mJy}_{\text {beam }}^{-1}, \sigma_{2400}=11 \mathrm{mJy}$ beam ${ }^{-1}$, and $\sigma_{4850}=11 \mathrm{mJy} \mathrm{beam}^{-1}$. Dashed contours are at $-2 \sigma$. Markers and lines are as in Fig. 1.

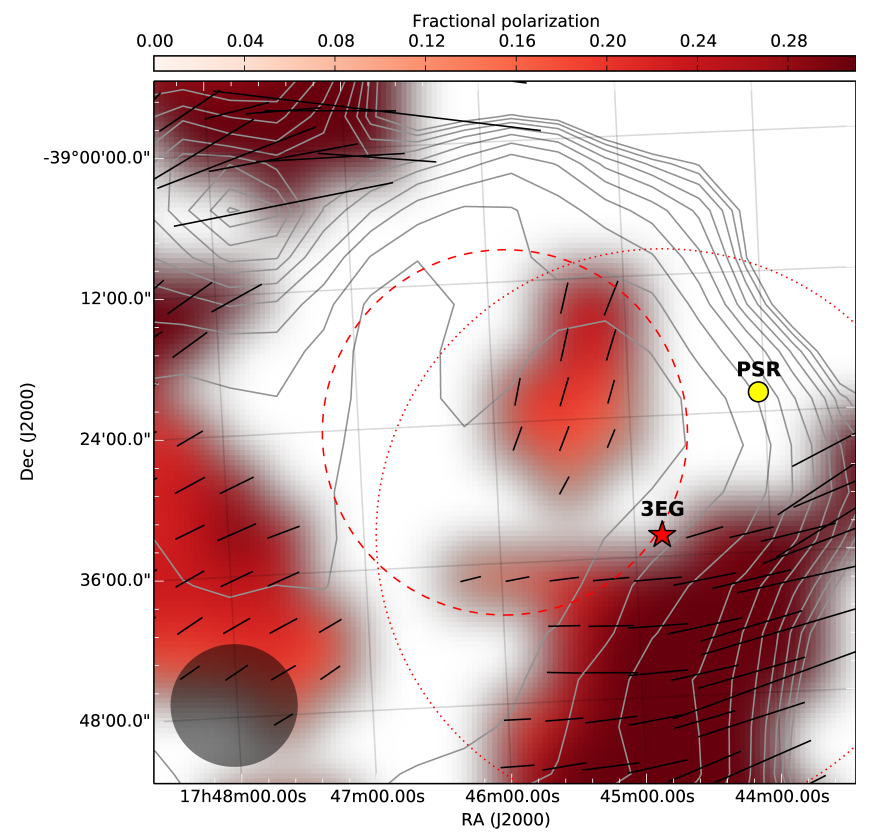

Fig. 3. Fractional polarization map obtained from the Parkes $2.4 \mathrm{GHz}$ Survey of the southern Galactic plane. E-vectors (perpendicular to the magnetic field) are also displayed for every pixel of the original maps. Pixel below $2 \sigma$ in the total intensity $\left(\sigma=11 \mathrm{mJybeam}^{-1}\right)$ or in the polarized intensity map $\left(\sigma=20 \mathrm{mJy}\right.$ beam $\left.^{-1}\right)$ were blanked.

The PMN survey was optimized for point source detection, consequently a low-pass filter was applied in the data reduction process (Griffith \& Wright 1993) with the effect to bias the flux densities of detected sources, mostly if extended, towards lower values. We note therefore that also PMN flux densities are likely to be underestimated.

\subsection{DECam}

In order to determine if the diffuse radio emission was a radio relic associated with a merging cluster of galaxies we observed the field in the optical during 4-7 Apr 2013 using DECam (DePoy et al. 2008) on the CTIO 4 m Blanco telescope. We observed the field in $g, r$, and $i$ to facilitate star-galaxy separation and potentially select cluster members. Exposures in $g, r$, and $i$

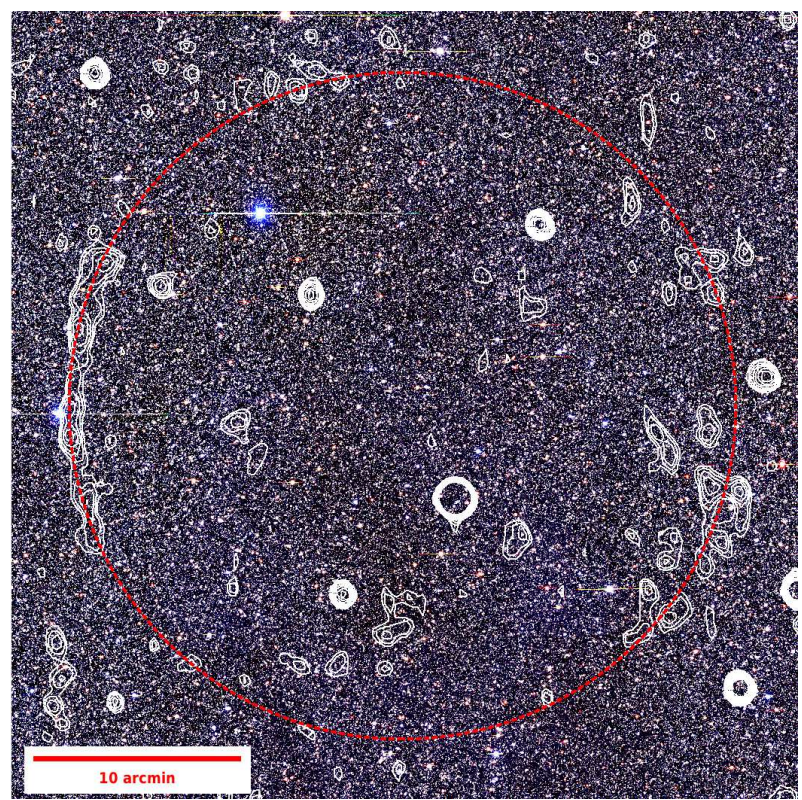

Fig. 4. DECam optical image ( $g, r$ and $i$ filters) of the target field. In red dashed-line the hypothetical size of the supernova remnant. Contours are from the NVSS survey $(1400 \mathrm{MHz})$ at $(2,2.75,3.5,4.25,5) \times \sigma$ with $\sigma=0.5$ mJy beam $^{-1}$.

were 100-200 s, dithered by $90^{\prime \prime}$ along each axis to cover the chip gaps. The total exposure times were $2000 \mathrm{~s}$ in $g, 2000 \mathrm{~s}$ in $r$, and $2623 \mathrm{~s}$ in $i$. These correspond to a $5 \sigma$ point source AB magnitude depths of 26.6 in $g, 26.1$ in $r$, and 25.8 in $i$. After accounting for Galactic extinction (Schlafly \& Finkbeiner 2011) the depths are 24.7 in $g, 24.8$ in $r$, and 24.8 in $i$. Using IRAF, all images were bias subtracted and flat-field corrected using nightly dome flats, then registered using msccmatch for alignment. Residual gradients across the CCDs were removed using imsurfit, and the resulting images were stacked using mscimatch to estimate the relative normalizations. The final image is shown in Fig. 4.

\subsection{Fermi-LAT}

To probe whether SNR G351.0-5.4 is a site of GCR acceleration, we searched for $\gamma$-ray emission at its location from the public 
Table 2. Radio observations.

\begin{tabular}{ccccccc}
\hline \hline Telescope & $\begin{array}{c}\text { Frequency } \\
(\mathrm{MHz})\end{array}$ & Resolution & $\begin{array}{c}\text { Largest scale } \\
(\operatorname{arcmin})\end{array}$ & $\begin{array}{c}\text { Flux density } \\
(\mathrm{mJy}) \\
\text { East }\end{array}$ & $\begin{array}{c}\text { Rms } \\
\left(\mathrm{mJy} \mathrm{beam}^{-1}\right)\end{array}$ \\
\hline GMRT & 325 & $141^{\prime \prime} \times 89^{\prime \prime}$ & $\simeq 32$ & 151 & 280 & 1.5 \\
VLA (NVSS) & 1400 & $45^{\prime \prime} \times 45^{\prime \prime}$ & $\simeq 16$ & 48 & 67 & 0.5 \\
Parkes 64 m & 2417 & $10^{\prime} .23 \times 10^{\prime} .62$ & - & $\sim 3000^{b}$ & 11 \\
Parkes 64 m (PMN) & 4850 & $4^{\prime} .9 \times 4^{\prime} .9$ & $\simeq 57^{a}$ & 367 & 162 & 11 \\
\hline
\end{tabular}

Notes. ${ }^{(a)}$ Although the PMN is a single dish survey, a limit on the largest scale is given by the filtering procedure applied during data reduction (Griffith \& Wright 1993) . ${ }^{(b)}$ The target emission is blended with the surrounding Galactic extended emission.

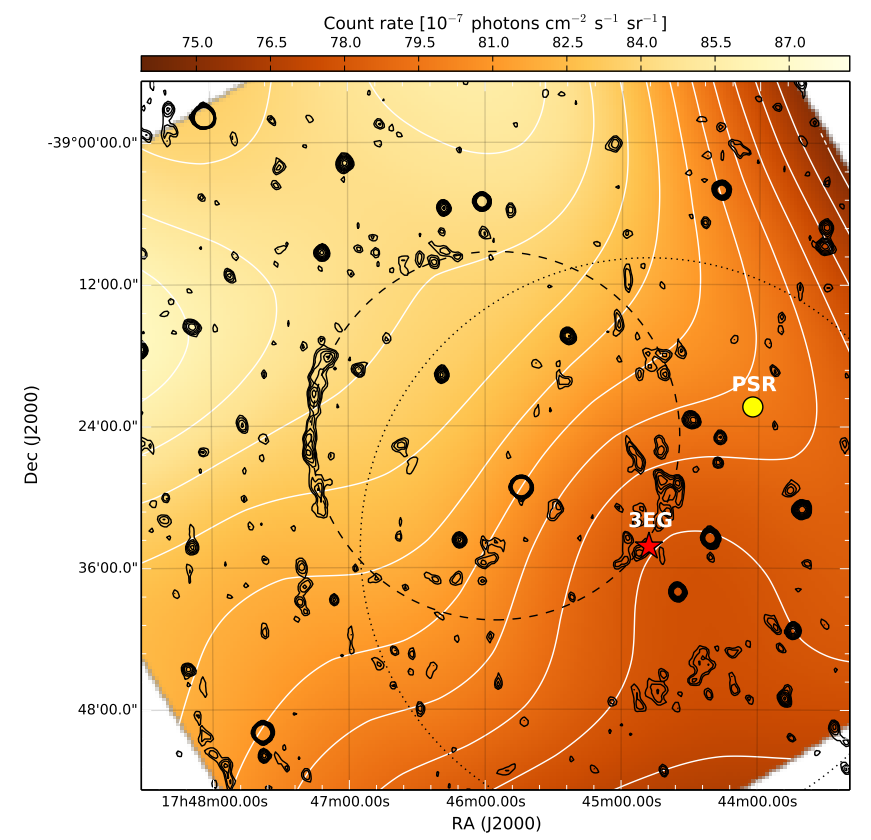

Fig. 5. Photon count from the Fermi-LAT observation in the energy band $1-500 \mathrm{GeV}$. The angular resolution $(68 \%$ containment radius) increases monotonically from $\sim 1^{\circ}$ to $\sim 0.2^{\circ}$ in the energy range 1-300 GeV (Ackermann et al. 2012). White contours follow the $\gamma$-ray emission. Black contours follow the NVSS 1.4 GHz emission (Fig. 2a). Markers and lines are as in Fig. 2a.

database of the Fermi-LAT. LAT on board the Fermi GammaRay Space Telescope is able to detect $\gamma$-rays with energies between $100 \mathrm{MeV}$ and $300 \mathrm{GeV}$ (Atwood et al. 2009). Data used in this work were obtained between 2008 August 4 and 2013 November 3 (Pass 7 Reprocessed Weekly Files), which are available from the Fermi Science Support Center ${ }^{3}$. We used the Fermi Science Tools "v9r23p1" package to reduce and analyse the data in the vicinity of SNR G351.0-5.4. The region of interest was defined as a square $\pm 3^{\circ}$ around the central point of SNR G351.05.4. From the Fermi-LAT data we extracted the $\gamma$-ray events for the region of interest in the energy range from 1 to $500 \mathrm{GeV}$. To calculate the flux we used the energy dependent point spread function of LAT (Ackermann et al. 2012). We did not considered energy events below $1 \mathrm{GeV}$ due to the very poor angular resolution. The image with the photon count is shown in Fig. 5.

\section{SNR G351.0-5.4}

The candidate SNR has a radius of $15^{\prime} .5$ with a centre located at $\alpha=17^{\mathrm{h}} 45^{\mathrm{m}} 55^{\mathrm{s}}, \delta=-39^{\circ} 24^{\mathrm{m}} 50^{\mathrm{s}}(\mathrm{J} 2000)$ or $l=351.06^{\circ}$, $b=-5.49^{\circ}$ (Galactic).

\footnotetext{
3 http://fermi.gsfc.nasa.gov/ssc/data/analysis/ software/
}

In Figs. 1 and 2 we present the four available radio images (325, 1400, 2417 and $4850 \mathrm{MHz})$ of SNR G351.0-5.4. In the high-resolution NVSS image the very narrow edge of the expanding shell is visible on the east side. On the west side a more disrupted edge is visible. Hints of emission come also from the north-east side of the shell and are mostly visible in the GMRT and PMN maps. The radio emission is globally not symmetric and mostly located on the expanding shell, features typical of old SNRs where the interaction with the ISM has already modified the symmetry present in the primary expansion stages (see e.g. Green 1986; Leahy \& Roger 1998, for IC443 and the Cygnus Loop respectively).

Given the radio morphology, another interpretation could be that the radio emission is a double radio relic generated by merging galaxy clusters producing shocks (Feretti et al. 2012). Relics are typically found in massive and merging systems with a hot intra-cluster medium $\left(10^{7}-10^{8} \mathrm{~K}\right)$ glowing in the X-ray. However, given the typical size of radio relics $(\sim 1 \mathrm{Mpc})$, the cluster should be at $z \sim 0.05$ and should have been detected by the ROSAT all sky survey although it is not. Furthermore, we find no evidence of significant galaxy over density associated with the radio emission in our optical observation (see Sect. 2.3). Given the limiting magnitudes of the optical images and that diffuse cluster radio emission has only been observed in rich massive clusters, we can confidently rule out any clusters associated with the radio emission below a redshift of 0.2 . Were the radio emission to be associated with a redshift greater then 0.2 , the projected physical scale of the emission would be $>16 \mathrm{Mpc}$. This is $>7 \sigma$ larger than the distribution of observed radio relics (Feretti et al. 2012). Thus it is highly unlikely that the diffuse radio emission is a radio relic associated with a merging galaxy cluster.

The extraction of a spectral index value is complicated because of the object's extension which could lower its flux density because of missing short baselines in interferometric observations and filtering in the single-dish case. This is clearly the case for the flux density extracted from the NVSS $1400 \mathrm{MHz}$ map, which in both the east and west sides of the source is lower than the flux density at 325 and $4850 \mathrm{MHz}$ implying a concave spectrum not compatible with synchrotron radiation.

\section{Discussion}

The radio emission of SNR G351.0-5.4 is coincident with an unknown EGRET $\gamma$-ray detection (3EG J1744-3934) which could be the consequence of the interaction between the expanding shell and the surrounding medium, e.g. molecular clouds. The $\gamma$-ray source 3EG J1744-3934 (Hartman et al. 1999) position, although having large errors $\left(r=0.66^{\circ}\right.$ is the $95 \%$ confidence area), is centred on the west side of SNR G351.0-5.4. The source is marked as a possibly extended/multiple source and has a flux 


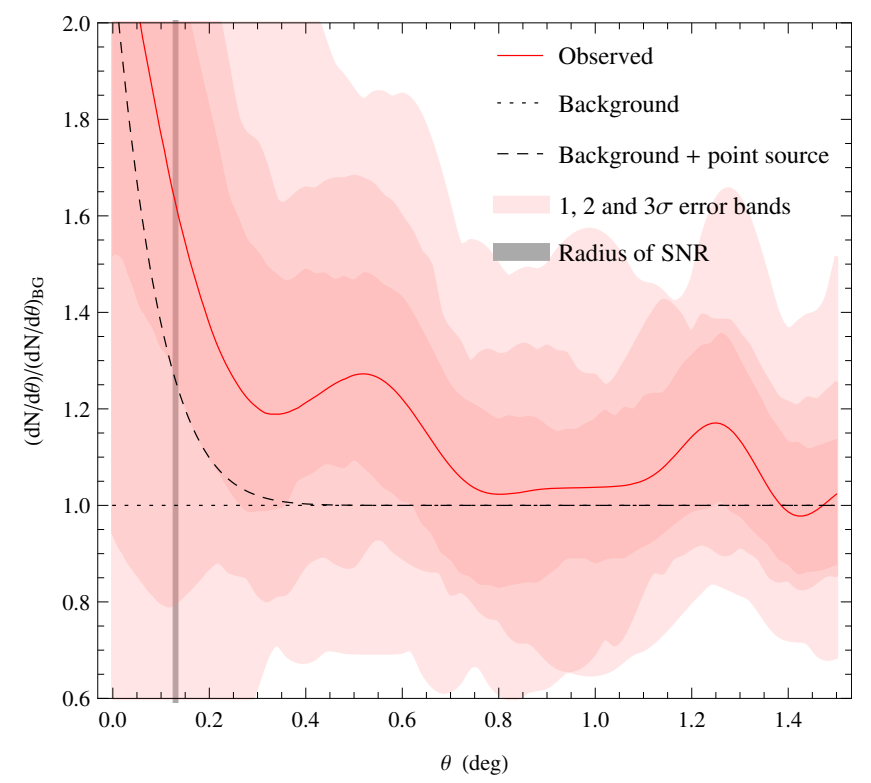

Fig. 6. Radial distribution of $\gamma$-ray events $(E>10 \mathrm{GeV})$ around the centre of the SNR. The red line denotes the ratio between the signal and the background estimated events (dotted line). Dashed line assumes a point source located at the centre of the SNR. A slightly spatially extended (confidence level $\sim 1.5 \sigma)$ feature is related with SNR. In addition, the two excesses can be seen at $0.5^{\circ}$ and $1.2^{\circ}$ from SNR probably related to the two Fermi-LAT point sources at those distances (Nolan et al. 2012).

of $F(>100 \mathrm{MeV})=17.1 \pm 3.5 \times 10^{-8}$ photon $\mathrm{cm}^{-2} \mathrm{~s}^{-1}$ and a spectral index $\gamma=2.42$. A new map of the $\gamma$-ray emission detected by Fermi-LAT in the energy range $1-500 \mathrm{GeV}$ in the area surrounding the nominal position of SNR G351.0-5.4 is reported in Fig. 5. We found no statistically significant $\gamma$-ray excess over the local background associated with SNR G351.0-5.4 in our study. The background is modelled as the averaged flux of region of interest, where the region $\left(<1^{\circ}\right)$ of the SNR and the Fermi-LAT point sources (Nolan et al. 2012) are excluded. In the region associated with the SNR, the limiting photon flux at energies $>1 \mathrm{GeV}$ is constrained to be $F(>1 \mathrm{GeV})<7.9 \times 10^{-9} \mathrm{~cm}^{-2} \mathrm{~s}^{-1}$. However, in the radial distribution of the $\gamma$-ray events around the centre of the SNR, a slightly spatially extended feature is detected with a confidence level of $\sim 1.5 \sigma$, see Fig. 6 .

$\mathrm{CO}$ and HI maps obtained by NANTEN telescope do not show any association between the SNR and possible molecular cloud systems (Y. Fukui, priv. comm.). If SNR G351.0-5.4 is indeed an old SNR, the $\gamma$-ray emission is absent because all CRs have escaped from the source, therefore energetic photons from neutral pion decay cannot be detected. This is in line with an absence of clouds along their path. If there were high density targets in the source surroundings, we could have seen $\mathrm{GeV}$ emission due to low-energy CRs (in the case of a target embedded in the source) or to high-energy CRs (in the case of a distant target) as in the case of the SNR W28 (Giuliani et al. 2010).

The pulsar PSR J1744-3922 is at a distance of $3.1 \mathrm{kpc}$, it was identified as a possible origin of the $\gamma$-ray emission for the source 3EG J1744-3934 (Hessels et al. 2005) and it is close to the target SNR $\left(\sim 23^{\prime}\right.$ from the SNR centre towards the west). A subsequent follow up with XMM showed no X-ray emission from the object and the source does not appear to be energetic enough to power the production of $\gamma$-rays (Faulkner et al. 2004).

The optical observation made with the DECam does not show any evidence of optical emission in any of the observed bands (see Fig. 4). The non-detection in the optical and in the $\gamma$-ray bands, together with the absence of $\mathrm{CO}$ and HI emission in the surroundings, the radio morphology and the low surface brightness, support the idea that SNR G351.0-5.4 must be an old SNR.

\section{Conclusions}

We report the discovery of an extended radio emission located in the proximity to the Galactic plane (Galactic latitude $b=$ $-5.5)$. The emission is visible in three radio surveys: NVSS at $1400 \mathrm{MHz}$, Parkes $64 \mathrm{~m}$ at $2417 \mathrm{MHz}$, and PMN at $4850 \mathrm{MHz}$. The source has been also detected by the GMRT at $325 \mathrm{MHz}$. The source has not been detected in the optical band. Given the source morphology, we classify it as the old shell of a Galactic supernova remnant which no longer emits in the optical and designate it SNR G351.0-5.4.

The presence of a SNR at these coordinates could also explain the unclassified EGRET $\gamma$-ray source 3EG J1744-3934 that may be the consequence of the interaction between particles escaping the remnant and the surrounding intra-galactic medium. However, deep analysis of the $\gamma$-ray data from Fermi-LAT did not show any significant excess in this region, no detection in $\mathrm{CO}$ or $\mathrm{HI}$ is also reported. In the radial distribution of the $\gamma$-ray events around the centre of the SNR, a slightly spatially extended excess is detected with a confidence level of $\sim 1.5 \sigma$.

Acknowledgements. We would like to thank Ishwara Chandra C. H. and the staff of the GMRT who made these observations possible. Based on observations obtained at Cerro Tololo Inter-American Observatory, a division of the National Optical Astronomy Observatories, which is operated by the Association of Universities for Research in Astronomy, Inc., under cooperative agreement with the National Science Foundation. A.H. acknowledges the European Social Fund for the grants MTT8, MTT60 and the European Regional Development Fund for the grant TK120.

\section{References}

Ackermann, M., Ajello, M., Albert, A., et al. 2012, ApJSS, 203, 4

Ackermann, M., Ajello, M., Allafort, A., et al. 2013, Science, 339, 807

Atwood, W. B., Abdo, A. A., Ackermann, M., et al. 2009, ApJ, 697, 1071

Berkhuijsen, E. M. 1984, A\&A, 140, 431

Cardillo, M., Tavani, M., Giuliani, A., et al. 2014, A\&A, 565, A74

Condon, J. J., Cotton, W. D., Greisen, E. W., et al. 1998, AJ, 115, 1693

DePoy, D. L., Abbott, T., Annis, J., et al. 2008, in Proc. SPIE, eds. I. S. McLean, \& M. M. Casali, 7014

Duncan, A. R., Haynes, R. F., Jones, K. L., \& Stewart, R. T. 2012, MNRAS, 291,

Faulkner, A. J., Stairs, I. H., Kramer, M., et al. 2004, MNRAS, 355, 147

Feretti, L., Giovannini, G., Govoni, F., \& Murgia, M. 2012, A\&ARv, 20, 54

Giuliani, A., Tavani, M., Bulgarelli, A., et al. 2010, A\&A, 516, L11

Green, D. A. 1986, MNRAS, 221, 473

Green, D. A. 2001, MNRAS, 326, 283

Green, D. A. 2009, Bull. Astron. Soc. India, 37, 45

Griffith, M. R., \& Wright, A. E. 1993, AJ, 105, 1666

Hartman, R. C., Bertsch, D. L., Bloom, S. D., et al. 1999, ApJSS, 123, 79

Hessels, J., Ransom, S., Roberts, M., et al. 2005, in Binary Radio Pulsars, ASP Conf. Ser., 328, 395

Katsuta, J., Uchiyama, Y., Tanaka, T., et al. 2012, ApJ, 752, 135

Leahy, D. A., \& Roger, R. S. 1998, ApJ, 505, 784

Malkov, M. A., \& Drury, L. O. 2001, Rep. Progr. Phys., 64, 429

Nolan, P. L., Abdo, A. A., Ackermann, M., et al. 2012, ApJSS, 199, 31

Noordam, J. E. 2004, in Proc. SPIE, 5489, 817

Offringa, A. R., van de Gronde, J. J., \& Roerdink, J. B. T. M. 2012, A\&A, 539, A95

Schlafly, E. F., \& Finkbeiner, D. P. 2011, ApJ, 737, 103

Tammann, G. A., Loeffler, W., \& Schroeder, A. 1994, ApJSS 92, 487

Tavani, M., Giuliani, A., Chen, A. W., et al. 2010, ApJ, 710, L151 